\title{
Immunogenicity Specimen Assessments Specimen Type
}

National Cancer Institute

\section{Source}

National Cancer Institute. Immunogenicity Specimen Assessments Specimen Type. NCI

Thesaurus. Code C117567.

The type of a material sample taken from a biological entity for immunogenicity specimen assessment testing. 